\title{
The hierarchical evolution of Brightest Cluster Galaxies: red galaxies in a young universe
}

\author{
Chiara Tonini \\ Centre for Astrophysics and Supercomputing, Swinburne University of Technology, VIC 3122, \\ Melbourne, Australia \\ ctonini@swin.edu.au
}

\begin{abstract}
We investigate the evolution of Brightest Cluster Galaxies (BCGs) from redshift $z \sim 1.6$ to $z=0$. We upgrade the hierarchical semi-analytic model of Croton et al. (2006) with a new spectro-photometric model that produces realistic galaxy spectra, making use of the Maraston (2005) stellar populations and a new recipe for the dust extinction. We compare the model predictions of the K-band luminosity evolution and the J-K, V-I and I-K colour evolution with a series of datasets, including Collins et al. (Nature, 2009) who argued that semianalytic models based on the Millennium simulation cannot reproduce the red colours and high luminosity of BCGs at $z>1$. We show instead that the model is well in range of the observed luminosity and correctly reproduces the colour evolution of BCGs in the whole redshift range up to $z \sim 1.6$. We argue that the success of the semi-analytic model is in large part due to the implementation of a more sophisticated spectro-photometric model. An analysis of the model BCGs shows an increase in mass by a factor $2-3$ since $z \sim 1$, and star formation activity down to low redshifts. While the consensus regarding BCGs is that they are passively evolving, we argue that this conclusion is affected by the degeneracy between star formation history and stellar population models used in SED-fitting, and by the inefficacy of toy-models of passive evolution to capture the complexity of real galaxies, especially those with rich merger histories like BCGs. Following this argument, we also show that in the semi-analytic model, the BCGs show a realistic mix of stellar populations, and that these stellar populations are mostly old. In addition, the age-redshift relation of the model BCGs follows that of the Universe, meaning that given their merger history and star formation history, the ageing of BCGs is always dominated by the ageing of their stellar populations. In a $\Lambda$ CDM Universe, we define such evolution as 'passive in the hierarchical sense'.
\end{abstract}

Keywords. Galaxies: evolution, fundamental parameters, star formation, photometry, stellar content - Galaxies: clusters: general

\section{The luminosity and colour evolution of BCGs}

We present the results for the luminosity and colour evolution of the Brightest Cluster Galaxies (BCGs), as predicted by a new version of the semi-analytic models of Croton et al. (2006), paired with Maraston (2005, M05) stellar population models. For a full description of the model and the results presented here, we defer the reader to the paper by Tonini et al. (2012), of which this proceeding is a short summary.

Fig. (1) shows the evolution of the observed-frame K-band luminosity for the model BCGs, compared with the sample discussed by Collins et al. (2010), and a number of samples from $z=0$ to $z \sim 1.6$ by Whiley et al. (2008), Stott et al. (2008), Brough et al. (2008), Lidman et al. (2012) and from GOODS (Daddi et al. 2007). This result shows that, contrary to the claim by Collins et al. (2009), the model BCGs are definitely bright enough. In other words, the stellar mass assembled by the model, constrained by the 


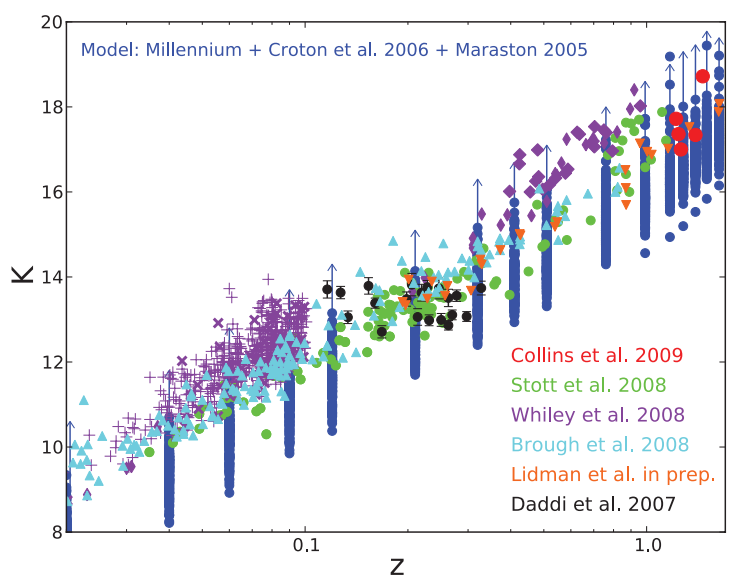

Figure 1. The observed-frame K-band luminosity evolution of the model BCGs (blue points), compared with a number of datasets: the sample discussed by Collins et al. (2010; red points), two samples by Whiley et al. (2008; Hubble and SDSS data, purple diamonds and crosses), a sample by Stott et al. (2008; green points), a sample by Brough et al. (2008; cyan triangles), a sample by Lidman et al. (orange triangles) and a sample from GOODS (Daddi et al. 2007, black squares).

hierarchical growth of structures, is adequate, and was not the reason for the discrepancies between models and data in the past. Up to $z \sim 1.6$, the extremely luminous galaxies that lie at the center of clusters are well represented in the model galaxy population. The new spectro-photometric model, quite successful in reproducing the high mass end of the luminosity function up to redshift 4 (see Henriques et al. 2011, Tonini et al. 2010), turned the discrepancy with BCGs observations upside-down. While it is reassuring to see that BCGs are no longer out of the model luminosity (and therefore mass) range, this result, however, highlights the degeneracy between the stellar population models and the details of the star formation history. We remind the reader that, amongst the available stellar population models, the M05-based model is the one with the highest K-band luminosity.

Fig. (2) shows the predicted evolution with redshift of the observed-frame $J-K$, $V-I$ and $I-K$ colors of the model BCGs bewteen $z \sim 0$ and $z \sim 1.6$, compared with the observations discussed in Collins et al. (2009), the data by Stott et al. (2008, 2010), the data by Lidman et al. (2012) and the Hubble and SDSS samples by Whiley et al. (2008). The colour evolution of the model BCGs agrees remarkably well with the data. As is expected from the definition of BCGs, the data lie at the very red end of the model colour distribution, across the whole redshift range. In all colors the model is also able to reproduce the steepening of the colour-redshift relation occurring at $z \sim 0.3$, but more data are needed to investigate this issue in more detail. More data would also be desirable to investigate the turnover of the model relation at $z>1$. This plot disproves the claim that hierarchical models are incapable of reproducing the very red $J-K$ colors of observed BCGs. Notice that this result did not require a re-calibration of the model parameters. For instance, we could have raised the cooling rate or cut off AGN feedback in an attempt to correct the failed semi-analytic+BC03 galaxies, but this would have affected the entire stellar mass function, not just the BCG population. As it is, the current semi-analytic model can accommodate extreme objects like BCGs at different redshifts with a more sophisticated spectro-photometric model. 

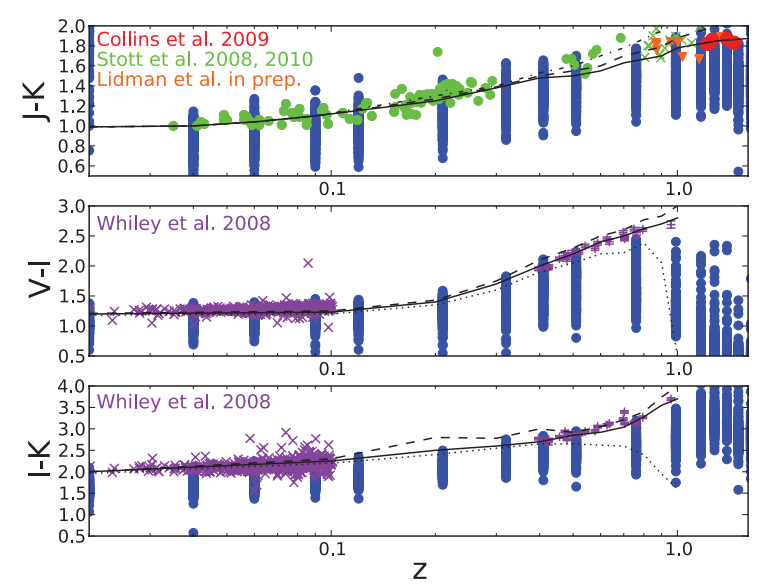

Figure 2. The color evolution of the model BCGs from redshift $z=0$ to $z \sim 1.6$ (in all panels the model galaxies are represented by blue points). Upper panel: $J-K$, data by Collins et al. (2010; red points), Stott et al. (2008, 2010; green points) and Lidman et al. (orange triangles). The lines represent toy models discussed in Collins et al. (2009) for single stellar populations (synthesized with the BC03 models), in the cases of no luminosity evolution (dot-dashed line), with passive evolution with formation redshifts of 5 (dashed line) and with formation redshift of 2 (solid line). Middle panel: $V-I$, data by Whiley et al. (2008; purple symbols. Lower panel: $I-K$, data by Whiley et al. (2008; purple symbols. In the middle and lower panels, the lines represent toy models discussed in Whiley et al. (2008) for single stellar populations (synthetised with the BC03 models), in the cases of passive evolution with formation redshifts of 1 (dotted line), formation redshift of 2 (solid line) and formation redshift of 5 (dashed line).

\section{The physical properties of BCGs}

As described in detail in Tonini et al. (2012), the model BCGs evolve following diverse star formation histories, with one feature in common: none of these galaxies is passively evolving, they all have some degree of residual star formation at $z=0$, and they are actively star forming at $z=1$. However, the BCG luminosity and colour evolution in a fully hierarchical assembly scenario, with the use of M05 stellar populations, is indistinguishable from a toy-model scenario of passive evolution with other stellar population models. The model BCGs are star forming and assembling a significant fraction of their mass (up to 50\%) after $z=1$ (see Tonini et al. 2012). However, how old are these galaxies? Or more accurately, how old are the stars that compose them? Do these galaxies follow a special evolutionary path in the hierarchical galaxy formation scenario?

Figure (3) shows the distribution of ages of the model BCGs as a function of redshift. We define the age of each BCG as the minimum age of a certain fraction of its stars, which corresponds to the lookback time of their formation. The contours represent the number of galaxies that have a certain age at a certain redshift. In the left panel we define the mass-weighted age of the BCG as the lookback time by which $50 \%$ of its stars were formed, while on the right panel we define the same quantity based on $90 \%$ of its stars. The black line in both plots shows the age of the Universe as a function of redshift. This figure reveals a number of things. First, the evolution of the model BCGs fans out from $z \sim 1$, showing a larger and larger variety in age as time goes by. This behaviour is driven by the differences in mass and environment, and is sustained by the ongoing star formation. This variety causes the colours of the model BCGs to scatter towards the blue. Second, most model BCGs are actually old. For most of them, at any given time the majority of their stars are of an age equal at least to half the age of the universe. Third, the BCGs population seems to grow old following the ageing of the universe itself. 

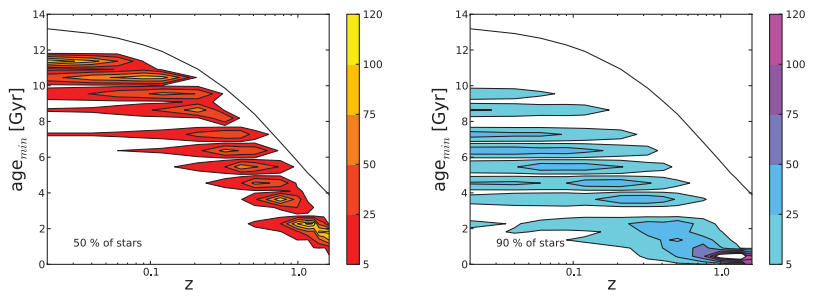

Figure 3. The distribution of ages of the model BCGs, as a function of redshift. We define the age of each BCG as the minimum age of a certain fraction of its stars, which corresponds to the lookback time of their formation. The contours represent the number of galaxies that have a certain age at a certain redshift. In the left panel we define the age of the BCG based on the oldest $50 \%$ of its stars, while on the right panel we define its age based on the oldest $90 \%$ of its stars. The black line in both plots shows the age of the Universe as a function of redshift.

There is a constant gap between the age of the oldest BCGs and the age of the universe (the black line in the plot), whether one considers the $50 \%$ or the $90 \%$ mark as an age indicator. Figure (3) means the following: the star formation in the model BCGs is not intense enough to offset their mass-weighted age since $z \sim 1.6$. There is not enough newly formed stellar mass to rejuvenate these objects, whose ageing is always dominated by the ageing of their stellar populations, regardless of where they were formed (main progenitor or satellites). For comparison, a truly actively star forming galaxy (like a spiral) would show a flatter age-redshift relation, which means that the fractional increase of stellar mass is dominated by new stellar populations (and not accretion of old stars), with a rate sufficiently high to lower the mass-weighted age. Since the ageing of the BCG is dominated by the ageing of its stellar populations (1 Gyr every Gyr), implying that the difference between the BCG age and the age of the Universe is constant, this represents an equivalent scenario to that of the ageing of a single stellar population in a passive evolution toy model. The difference is that we are now considering not just one stellar population, but all the populations that end up forming the BCG, i.e. we are looking at the collective ageing of the stellar component in the merger tree. We define this behaviour as 'passive evolution in the hierarchical sense'.

\section{References}

Brough, S., Couch, W. J., Collins, C. A., Jarrett, T., Burke, D. J., \& Mann, R. G. 2008, MNRAS, 385,103

Collins, C. A., et al. 2009, Nature, 458, 603

Croton, D. J., et al. 2006, MNRAS, 365, 11

Daddi, E., et al. 2007, ApJ, 670, 156

Maraston, C. 2005, MNRAS, 362, 799

Stott, J. P., Edge, A. C., Smith, G. P., Swinbank, A. M., \& Ebeling, H. 2008, MNRAS, 384, 1502

Stott, J. P., et al. 2010, ApJ, 718, 23

Tonini, C., Maraston, C., Devriendt, J., Thomas, D., \& Silk, J. 2009, MNRAS, 396, 36

Tonini, C., Maraston, C., Thomas, D., Devriendt, J., \& Silk, J. 2010, MNRAS, 403, 1749

Tonini, C., Maraston, C., Ziegler, B., Böhm, A., Thomas, D., Devriendt, J., \& Silk, J. 2011, MNRAS, 415, 811

Tonini, C., Bernyk, M., Croton, D., Maraston, C., \& Thomas, D. 2012, ApJ, 759, 43

Whiley, I. M., et al. 2008, MNRAS, 387, 1253 